\title{
Engineered Reversal of Function in Glycolytic Yeast Promoters
}

Rajkumar, Arun S.; Özdemir, Emre; Lis, Alicia V.; Schneider, Konstantin; Qin, Jiufu; Jensen, Michael K.; Keasling, Jay D.

\section{Published in:}

ACS Synthetic Biology

Link to article, DOI:

10.1021/acssynbio.9b00027

Publication date:

2019

Document Version

Publisher's PDF, also known as Version of record

Link back to DTU Orbit

Citation $(A P A)$ :

Rajkumar, A. S., Özdemir, E., Lis, A. V., Schneider, K., Qin, J., Jensen, M. K., \& Keasling, J. D. (2019).

Engineered Reversal of Function in Glycolytic Yeast Promoters. ACS Synthetic Biology, 8(6), 1462-1468. https://doi.org/10.1021/acssynbio.9b00027

\section{General rights}

Copyright and moral rights for the publications made accessible in the public portal are retained by the authors and/or other copyright owners and it is a condition of accessing publications that users recognise and abide by the legal requirements associated with these rights.

- Users may download and print one copy of any publication from the public portal for the purpose of private study or research.

- You may not further distribute the material or use it for any profit-making activity or commercial gain

- You may freely distribute the URL identifying the publication in the public portal 


\title{
Engineered Reversal of Function in Glycolytic Yeast Promoters
}

\author{
Arun S. Rajkumar, ${ }^{\dagger, \#}$ Emre Özdemir, ${ }^{\dagger}$ Alicia V. Lis, ${ }^{\dagger}$ Konstantin Schneider, ${ }^{\dagger}$ Jiufu Qin, ${ }^{\dagger}$ \\ Michael K. Jensen, ${ }^{*} \dagger$ and Jay D. Keasling ${ }^{\dagger,+, \S, \|, \perp}$ \\ ${ }^{\dagger}$ Novo Nordisk Foundation Center for Biosustainability, Technical University of Denmark, DK-2800 Kgs. Lyngby, Denmark \\ ${ }^{\ddagger}$ Joint BioEnergy Institute, Emeryville, California 94608, United States \\ ${ }^{\S}$ Biological Systems and Engineering Division, Lawrence Berkeley National Laboratory, Berkeley, California 94704, United States \\ "Department of Chemical and Biomolecular Engineering \& Department of Bioengineering, University of California, Berkeley, \\ California 94720-1462, United States \\ ${ }^{\perp}$ Center for Synthetic Biochemistry, Institute for Synthetic Biology, Shenzhen Institutes of Advanced Technologies, Shenzhen, \\ China
}

\section{Supporting Information}

\begin{abstract}
Promoters are key components of cell factory design, allowing precise expression of genes in a heterologous pathway. Several commonly used promoters in yeast cell factories belong to glycolytic genes, highly expressed in actively growing yeast when glucose is used as a carbon source. However, their expression can be suboptimal when alternate carbon sources are used, or if there is a need to decouple growth from production. Hence, there is a need for alternate promoters for different carbon sources and production schemes. In this work, we demonstrate a reversal of regulatory function in two glycolytic yeast promoters by replacing glycolytic regulatory elements with ones induced by the diauxic shift. We observe a shift in induction from glucose-rich to glucose-poor medium without loss of regulatory activity, and strong ethanol induction. Applications of these promoters were validated for expression of the vanillin biosynthetic pathway, reaching production of vanillin comparable to pathway designs using strong constitutive promoters.
\end{abstract}

KEYWORDS: transcriptome, synthetic promoter, glycolysis, gluconeogenesis, vanillin

$\mathrm{T}$ he budding yeast Saccharomyces cerevisiae is a popular cell factory platform, with several genetic and molecular tools available to facilitate the production of compounds of interest for the chemical and pharmaceutical industry. The synthesis of increasingly complex molecules, in turn, require successful construction of large and complex heterologous pathways, ${ }^{1-3}$ which impose an added metabolic burden by competing for required cellular resources with native metabolism. Competition for a common pool of ATP, cofactors, coenzymes and precursors between native and heterologous pathways can adversely affect growth, lengthen fermentation, and decrease product titers and yields. ${ }^{4-6}$ One way to avoid this problem is to activate the production pathway after the major growth phase. For a typical process with a fermentable carbon source, a good regulatory trigger would be the diauxic shift following fermentation. ${ }^{7,8}$ However, most promoters commonly used for heterologous pathway construction are glycolytic (PGK1pr, TPI1pr, or TDH3pr) or constitutive (TEF1pr), and lack the desired induction properties. Native yeast promoters have been identified for gene expression under these conditions and used for production, ${ }^{7-11}$ yet it is unclear if sufficient native promoters with suitable properties exist to be used for large biosynthetic pathways. Therefore, using synthetic promoters with optimal regulatory output at the diauxic shift would be beneficial for cell factory design. This can be achieved by hybrid promoter fusion or mutagenesis, ${ }^{12,13}$ but the level of control over regulatory signals or expression levels using these approaches may not be sufficient. In previous work, we developed a promoter engineering workflow to engineer yeast promoters responsive to any environmental condition given transcriptome or transcription factor binding site (TFBS) data, and functional genomics for the condition of interest, if available. ${ }^{14}$ Other studies have also made use of expression data in a similar manner to engineer synthetic promoters and biosensors. $^{15,16}$ However, generally most work on synthetic Saccharomyces promoters does not focus on rationally engineering native regulatory elements to generate defined regulatory properties, as has been done in other yeasts. Having used this workflow to make promoters inducible by low extracellular $\mathrm{pH},{ }^{14}$ we now use the same workflow to design promoters inducible by glucose starvation and alternating carbon sources.

Using S. cerevisiae CEN.PK 113-7D transcriptome data generated during the lag ( $10 \%$ glucose consumption, low

Received: January 22, 2019

Published: May 3, 2019 


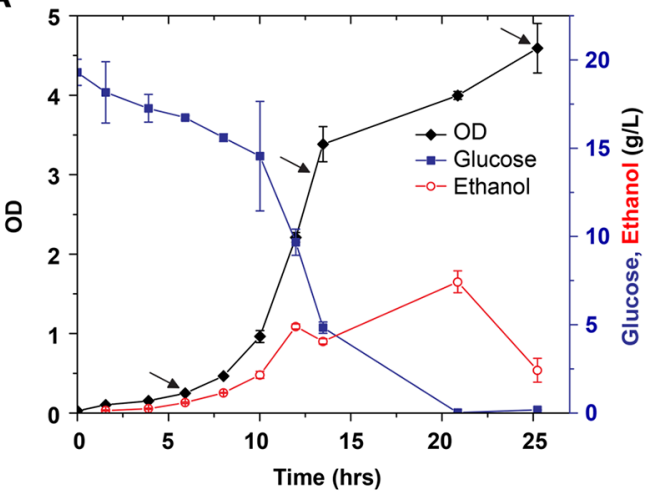

D

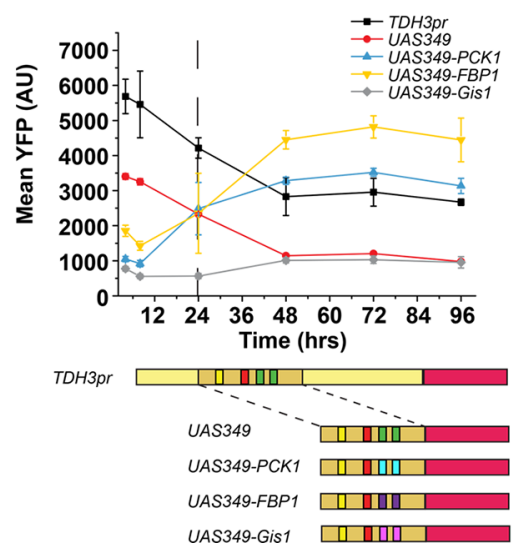

B

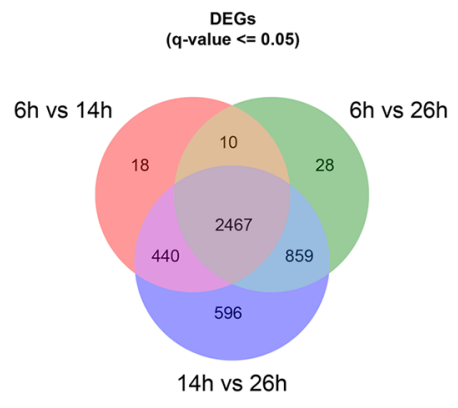

C

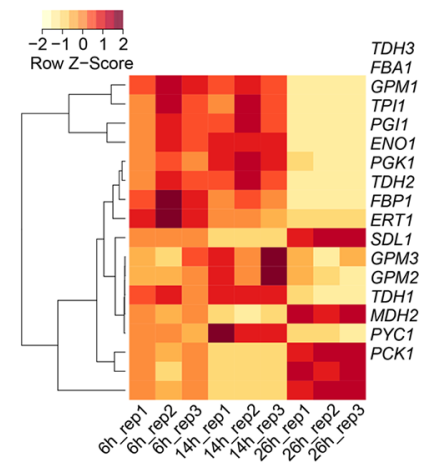

E

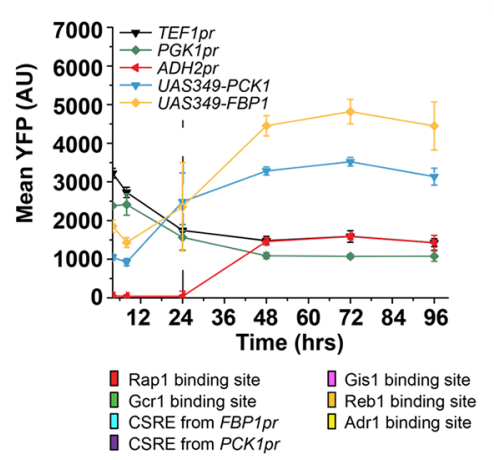

$\mathbf{F}$

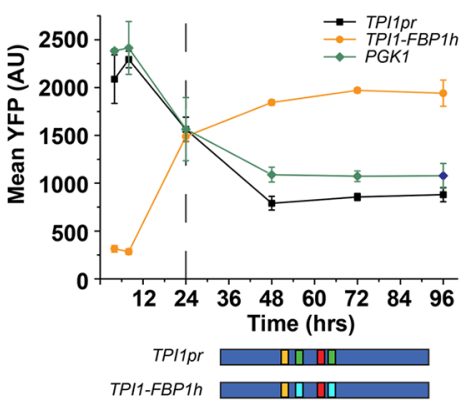

Figure 1. Yeast transcriptome analysis during lag, mid-exponential, and post-exponential phases, and engineering of diauxic responses in glycolytic promoters. (A) Growth profile of S. cerevisiae CEN.PK grown in Delft medium (black), and the corresponding glucose (dark blue) and ethanol (red) concentrations. Black arrows indicate sampling time-points $(6,14$, and $26 \mathrm{~h})$ for RNaseq analysis. Measurements are mean \pm SEM from three biological replicates. [SEM = standard error of the mean.] (B) Venn diagram showing the numbers of significantly differentially expressed genes (DEGs) from the three-way comparison between sampling points as indicated in panel (A). (C) Heat map of triplicate expression profiles of 17 DEGs following the diauxic shift (14 h vs $26 \mathrm{~h}$ ) under GO term for gluconeogenesis (GO:0006094). (D) A 200 bp UAS from TDH3pr was fused upstream of the TDH3 core promoter, creating UAS-349, a relatively strong promoter exhibiting the same trends as intact TDH3pr with its output decreasing over time once glucose is depleted. Replacing its Gcrl binding sites with CSREs from gluconeogenic genes (UAS349-PCK1, UAS349FBP1) reverses the induction pattern, with an output stronger than the native TDH3pr once glucose is depleted. Replacing Gcr1 sites with Gis1 sites (UAS349-Gis1) does not have the same effect. (E) The synthetic gluconeogenic promoters are stronger than $A D H 2 p r$, a native promoter strongly derepressed on glucose starvation, and the commonly used TEF1pr and PGK1pr. (F) Replacing Gcrl sites in TPI 1p with CSREs from FBP1pr into TPI1pr cause reversal of glucose-dependent induction, even though Gcrl sites in TPIlpr are differently spaced and oriented. For panels (D) $-(\mathrm{F})$, the dashed line indicates the time point where glucose was no longer detected, as described in the Methods section. All measurements in panels (D) $-(F)$ represents the mean \pm SEM from three biological replicates. AU = arbitrary units. At the bottom of panels (D) (F), illustrations outline the schematics of the promoter engineering. The sequences of the binding sites are listed in Table S6 in the Supporting Information, and all promoter sequences are listed in Table S7 in the Supporting Information.

ethanol production), mid-exponential (75\% glucose consumed, increasing ethanol production), and post-exponential phases (>99\% glucose consumed, start of ethanol consumption), we identified genes with the desired expression profile: strong induction upon glucose depletion (see Figure 1A). Induction under these conditions involves transcriptional activators such as Cat8, Sip4, Rds2, and Adr1 (Table 1), clustered in carbon source response elements (CSREs) in promoters of interest. ${ }^{17-21}$ Therefore, CSREs are relevant regulatory elements to ensure that promoters are activated at the diauxic shift and remain activated past this shift. Transcriptome analysis revealed 4418 differentially expressed genes (DEGs) from the 3-way comparison, with the majority being induced at, or following, glucose depletion and the start of diauxic shift ( $26 \mathrm{~h}$, as compared to $14 \mathrm{~h}$ ), including 17 out of 19 genes (excluding ENO2 and PYC2) associated with gluconeogenesis (term GO:0006094) (see Figures 1B and 1C, as well as Table $\mathrm{S} 1$ in the Supporting Information).

Next, we scanned the promoters for clustered TFBSs in CSREs. However, reported sequences for their binding sites contain palindromic or inverted repeats, as well as stretches of ambiguous bases. For further confirmation, we analyzed the discovered TFBSs for overlap with CSREs reported in the literature (see Table 1, as well as Table S2 in the Supporting Information). From this analysis, we selected CSREs from $P C K 1$ and FBP1 as candidate elements for synthetic promoter engineering based on three criteria: (i) promoters had a strong induction and appeared in both gene ontology mappings (see Table 1, as well as Figure S1 in the Supporting Information); (ii) the CSREs have been experimentally validated in the past in multiple studies; ${ }^{11,22}$ and (iii) while other genes had a higher fold-induction, e.g., MLS1, the CSREs from FBP1 and $P C K 1$ promoters also contained the most overlapping TFBSs. 
Table 1. Genes Associated with Carbon Metabolism Upregulated between the Log Phase and Diauxic Shift

\begin{tabular}{|c|c|c|c|}
\hline gene & $\begin{array}{l}\text { fold- } \\
\text { induction }\end{array}$ & binding sites in CSREs & $\begin{array}{c}\text { references for binding } \\
\text { sites }\end{array}$ \\
\hline \multicolumn{4}{|c|}{ Annotated "Gluconeogenesis" by Gene Ontology } \\
\hline PCK1 & 472.97 & Adr1, Cat8, Sip4, Rds2 & $18,20,23,24$ \\
\hline FBP1 & 52.67 & Adr1, Cat8, Rds2 & $18,20,24$ \\
\hline $\mathrm{MDH} 2$ & 6.55 & Adr1, Cat8, Rds2 & $18,20,23$ \\
\hline PYC1 & 1.87 & Cat8 & 18,20 \\
\hline GPM2 & 1.74 & none & \\
\hline PYC2 & 1.46 & none & \\
\hline \multicolumn{4}{|c|}{ Annotated "Carbon Metabolic Process" by GO_slim } \\
\hline MLS1 & 650.68 & Adr1, Cat8, Rds2 & $18,20,24$ \\
\hline PCK1 & 472.97 & Adr1, Cat8, Sip4, Rds2 & $18,20,23,24$ \\
\hline ICL1 & 145.86 & Cat8, Sip4 & $18,19,20,23$ \\
\hline YIG1 & 129.24 & Adr1,Cat8 & 23 \\
\hline FBP1 & 52.67 & Adr1, Cat8, Rds2 & $18,20,24$ \\
\hline$M A L 12$ & 28.48 & Cat8 & 23 \\
\hline MAL11 & 29.81 & Cat8 & 23 \\
\hline CAT8 & 22.31 & none & \\
\hline$M A L 32$ & 16.89 & none & \\
\hline GAL3 & 13.67 & Cat8 & 23 \\
\hline $\mathrm{MDH} 2$ & 6.55 & Adr1, Cat8, Rds2 & $18,20,23$ \\
\hline$D A K 2$ & 6.67 & none & \\
\hline PYC1 & 1.87 & Cat8 & 18,20 \\
\hline \multicolumn{4}{|c|}{ Fold-Induction of Other Native Promoters } \\
\hline$A D H 2$ & 10.93 & Cat8, Sip4 & $18,19,21$ \\
\hline TDH3 & 0.163 & none & \\
\hline TEF1 & 0.762 & none & \\
\hline$P G K 1$ & 0.192 & Adr1 & 23 \\
\hline TPI1 & 0.0732 & none & \\
\hline
\end{tabular}

This potential redundancy of function would allow for more reliable induction and ensure that the promoter activity would remain activated past the diauxic shift. ${ }^{20,21,23}$ Finally, since native $P C K 1$ and $F B P 1$ promoters have relative high expression levels during the early exponential phase (see Table S1 in the Supporting Information), these native promoters did not themselves have desired gluconeogenic responses.

These CSREs were used to engineer a $200 \mathrm{bp}$ upstream activating sequence (UAS) from the glycolytic TDH3 promoter (TDH3pr). We chose this UAS to test the reversal of regulatory function by TFBS exchange, because its main regulatory elements are well-annotated and confirmed by ChIP-chip studies (Figures 1D and 1E). ${ }^{24}$ In particular, this UAS contains all the Gcrl binding sites in TDH3pr, and this TF is a key regulator of glycolytic genes. ${ }^{25,26}$ It also contains TFBSs for Rap1, which is a TF that we have previously shown to increase promoter strength. ${ }^{12}$ It was therefore in the interest of maintaining high expression under relevant conditions to retain these parts of the UAS architecture, rather than swap the TDH3pr UAS with that of PCK1pr or FBP1pr. YFP reporter assays revealed that the UAS fused to the $T D H 3$ core promoter (UAS349) retained $60 \%$ of the activity of TDH3pr (see Figure $1 D$, as well as Figures S2 and S3 in the Supporting Information). Replacing both Gcr1 sites and surrounding neutral sequence with CSREs from either PCK1pr or FBP1pr to yield promoters UAS349-PCK1 and UAS349-FBP1 (Figure 1D) did indeed shift the induction trigger of the UAS from a glucose-rich medium to a glucose-depleted medium, with the induction of UAS349-PCK1 and UAS349-FBP1 triggered by glucose depletion, after $\sim 24 \mathrm{~h}$ of culture. Importantly, both promoters had a stronger output than not only UAS349 and but also $T D H 3 p r$ - one of the strongest yeast promoters in use-which was sustained as long as glucose was not replenished. The replacement of Gcrl sites with CSREs was sufficient and necessary for the induction. When they were exchanged with Gis1 (a TF implicated in upregulating genes at the stationary phase) binding sites instead (UAS349-Gis1), ${ }^{27}$ the promoter did not induce upon glucose starvation and remained weak throughout the assay period (Figure 1D). Ultimately, UAS349-PCK1 and UAS349-FBP1 were up to 3fold stronger than commonly used constitutive promoters over long time periods (Figure 1E). A stronger switch in induction was achieved when the Gcrl site exchange was made for the triose phosphate isomerase promoter (TPI1pr) with CSREs from FBP1pr (TPI1-FBP1h; see Figure 1F, as well as Figures S2 and S3). ${ }^{28}$ Although the orientation, spacing, and sequence context of Gcrl sites in both TDH3pr and TPIlpr were different, we observed near-identical induction behavior. It is likely the change in induction is independent of the local context of Gcrl sites, provided that no other neighboring TFBSs are modified (as was the case for TDH3pr and TPIIpr), and that their substitution could be applied to engineer induction switching in any glycolytic promoter. Finally, the synthetic promoters are stronger when grown on ethanol as a carbon source (Figure S4 in the Supporting Information). The lack of glucose repression, and the higher levels of ethanol (23 -fold), compared to those built up during the growth on glucose, probably induce them to higher levels.

To demonstrate the usefulness of our synthetic promoters, we applied them for the design of yeast cell factories for vanillin- $\beta$-glucoside biosynthesis (Figure $2 \mathrm{~A}$ ). ${ }^{29}$ The choice of product was motivated by the fact that actual vanillin- $\beta$ glucoside synthesis, when using glycolytic promoters, occurs in the ethanol phase. ${ }^{30}$ In existing cell factories and their fermentations, $\sim 70 \%$ of the carbon ends up as toxic intermediates like protocatechuic acid (PCA), continuously produced and largely secreted through both glucose and ethanol phases. ${ }^{31,32}$ We used UAS349-PCK1 and UAS349FBP1 instead of constitutive promoters only for the enzymes that convert PCA to vanillin (ACAR and EntD (see Figure 2A, as well as Figure $\mathrm{S} 5$ in the Supporting Information), expecting improved PCA conversion, faster growth, and ultimately similar or higher extracellular vanillin- $\beta$-glucoside production (Figure 2A). Compared to the existing pathway design relying on the use of constitutive (TEF1pr) and glycolytic (PGK1pr) promoters $(\mathrm{C}-\mathrm{VG})$ alone, ${ }^{30}$ the use of synthetic gluconeogenic promoters to control expression of the vanillin- $\beta$-glucoside biosynthetic pathway (strains ScASR.V001 and ScASR.V003) supported production of vanillin- $\beta$-glucoside to similar levels as the C-VG strain, following a $65 \mathrm{~h}$ fermentation (Figure 2B). Similarly, the growth and glucose consumption rates were increased, and conversion of PCA toward protocatechuic aldehyde (PAL) was also improved in the engineered strains, compared to C-VG (see Figures 2C and 2D, as well as Figures S6-S8 in the Supporting Information). Ultimately, ScASR.V003 produced a higher amount of vanillin- $\beta$-glucoside as a percentage of the total heterologous extracellular metabolites, suggesting that the CSRE from FBP1pr has the best performance for engineering gluconeogenic promoters for practical applications (see Figure 2D, as well as Figure S8). We can envisage further improving PCA conversion by placing the enzyme synthesizing it from 3-dehydroshikimate (3-DSD) under the control of another synthetic promoter responsive to 
A

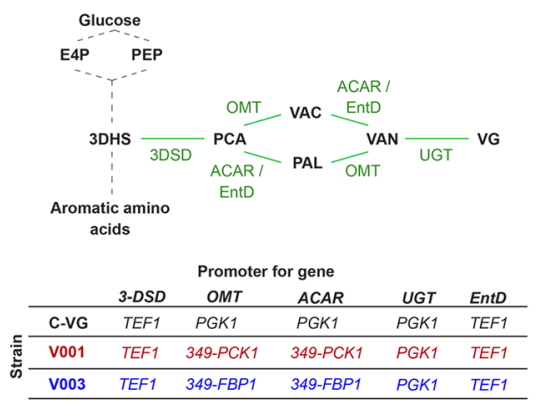

C

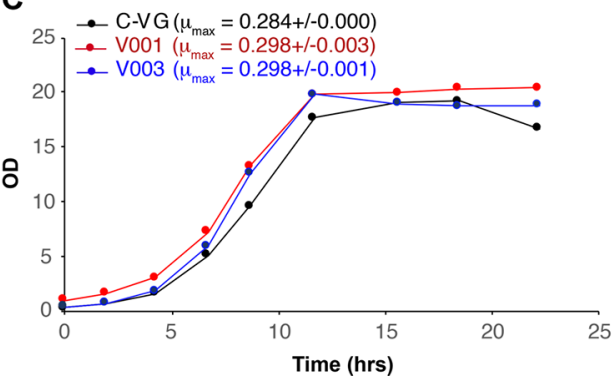

B
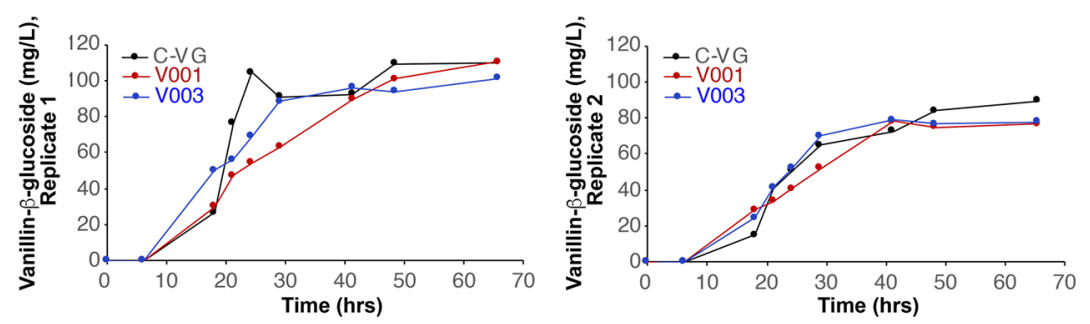

D
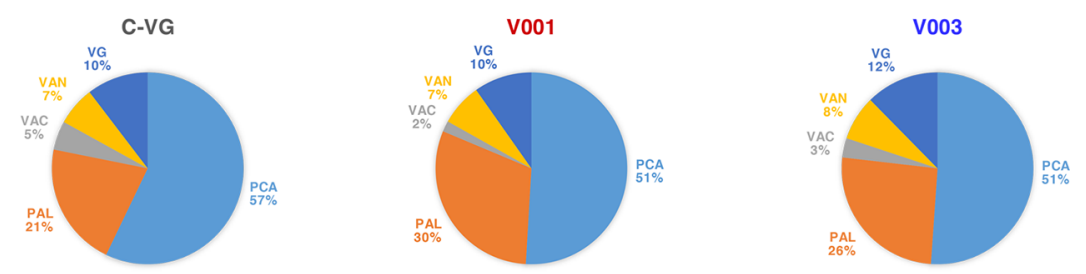

Figure 2. Benchmarking vanillin- $\beta$-glucoside production in yeast using synthetic gluconeogenic promoters. (A) Vanillin- $\beta$-glucoside pathway overview (left) and layout of promoter usage in control C-VG strain, ${ }^{30}$ and strains ScASR.V001 and ASR.V003 engineered with using synthetic gluconeogenic promoters (right). Starting from the shikimate pathway, the vanillin- $\beta$-glucoside pathway is marked in green. (B) Vanillin- $\beta$ glucoside production during $65 \mathrm{~h}$ cultivations in 5 -mL-deep well plates. Data represent two independent biological replicates samples. (C) Representative growth profiles of vanillin- $\beta$-glucoside production strains C-VG, ScASR.V001, and ScASR.V003 cultivated in duplicates in 250 -mL shake flasks. Mean growth rates \pm the standard deviation (SD) from duplicate measurement are inserted (D) Pie charts illustrating average relative distribution of the pathway products for each strain, as a percentage of the total products of the vanillin- $\beta$-glucoside pathway. The average metabolite distribution is based on two biological replicates sampled at the end of $65 \mathrm{~h}$ fermentations (see panel (B), as well as Figure S7). [Abbreviations: PCA, protocatechuic acid; PAL, protocatechuic aldelyde; VAC, vanillic acid; VAN, vanillin; and VG, vanillin- $\beta$-glucoside.] For panels (B) - (D), growth curves, growth rates, and metabolite profiles from individual cultivations can be found in Figures S6-S8.

glucose depletion; the current choice (TEF1pr) still shows some decreased activity under glucose depletion or high ethanol (see Figure 1D, as well as Figures S1 and S4). In this way, the entire pathway would only be activated when sufficient biomass has accumulated and PCA is given no time to accumulate and be secreted, as seen in the strain designs tested here. Testing different CSREs in synthetic promoters would also allow us to select more promoters for every gene in the pathway in future configurations. Ultimately, it may be advantageous to have a combination of CSREs (native or synthetic) and glycolytic TFBSs to modulate expression across glucose concentrations. Such a configuration would be beneficial in fermentations where glucose levels are low or periodically replenished, such as a fed-batch cultivation.

The promoters that have been engineered and put to use in this study reaffirm our promoter engineering strategy, ${ }^{14}$ which does not require altered transcription factor expression, or orthogonal regulatory systems. ${ }^{33-35}$ We can foresee such synthetic promoters being designed as needed, or used off-theshelf in other applications where the bulk of products are made during ethanol consumption phases, when a product or enzyme is toxic to the cell and decoupling production from growth is potentially beneficial, or where multiple carbon sources are being used. ${ }^{30,36,37}$ The last application is of interest for our promoters, because they also show strong induction when grown in ethanol (see Figure S4). Our current promoters were mainly engineered by replacing TFBSs in known promoter architectures, but we expect future engineering efforts to more explicitly and optimally space regulatory elements in both the upstream activation/repressive elements and core promoters. ${ }^{38-41}$ Since promoters for cell factories may not require induction at the diauxic phase, future engineering efforts will focus on finding an optimal balance and configuration of glycolytic, diauxic, and gluconeogenic transcription factors and their binding sites to achieve constitutively high expression levels ultimately supporting the rational design of next-generation, high-performance yeast cell factories.

\section{MATERIALS AND METHODS}

Reporter Strain Construction. S. cerevisiae CEN.PK 113$7 \mathrm{D}$ was used as background strain for all experiments. Promoters were amplified from genomic DNA or gBlocks (IDT) using Phusion Master Mix with HF buffer (ThermoFisher). YFP and resistance markers were amplified from plasmid pASR0130. ${ }^{12}$ YFP expression cassettes with different promoters were cloned into the EasyClone site XII-4 using in vivo assembly by homologous recombination. In brief, 500 pmol of the relevant promoter, YFP, the VPS13 terminator, and $\operatorname{kanMX}$ cassette for G418 resistance marker were transformed to yeast with $500 \mathrm{bp}$ of homology to XII-4 on either end by the lithium acetate method onto YPD agar with $350 \mathrm{ng} / \mu \mathrm{L}$ G418. Parts used in assembly had $50 \mathrm{bp}$ homology with adjacent parts added by PCR. G418-resistant colonies were screened by multiplex colony $\mathrm{PCR}$ as previously described, ${ }^{42}$ checking for integration and assembly at the correct locus. Colonies with sequence-verified reporter constructs were streaked out on YPD agar, regrown in YPD with $200 \mathrm{ng} / \mu \mathrm{L}$ G418 and preserved as glycerol stocks. All primers used for strain construction are listed in Table S3, and all constructed strains are listed in Table S4. 
YFP Reporter Assays. Reporter strains, as well as the background strain, were grown overnight in synthetic drop-out medium minus leucine (SD-leu, Sigma) containing $1.1 \mathrm{~g} / \mathrm{L}$ monosodium glutamate as a carbon source and $200 \mathrm{ng} / \mu \mathrm{L}$ G418 where appropriate. These were diluted to an OD of $\sim 0.02$ in minimal Delft medium, ${ }^{43} \mathrm{pH} 6$ and grown at $30^{\circ} \mathrm{C}$ in deep-well microtiter plates with $300 \mathrm{rpm}$ agitation. The culture was sampled at $4,8,24,48,72$, and $96 \mathrm{~h}$ for measuring YFP fluorescence by flow cytometry. Cells were suitably diluted in phosphate-buffered saline (PBS) (Life Technologies) and their fluorescence measured on an LSRFortessa flow cytometer (BD Biosciences) equipped with an HTS module for sampling. YFP fluorescence was measured with $488 \mathrm{~nm}$ excitation and $530 \mathrm{~nm}$ emission. A total of 10000 events per sample were acquired using FACSDiva software, and the resulting FCS files were analyzed using the FCSExtract utility (http://research.stowers. $\mathrm{org} / \mathrm{mcm} / \mathrm{efg} /$ ScientificSoftware/Utility/FCSExtract/index. htm), R scripts and Origin 9.1 (OriginLab) to extract the mean population fluorescence. Alternatively, the cells sampled at the same time points and diluted in PBS had their OD and YFP fluorescence (488 nm excitation and $527 \mathrm{~nm}$ emission) measured on a SynergyMX microtiter plate reader using clear-bottomed black microtiter plates (Thermofisher), and fluorescence values normalized to sample OD following the subtraction of background fluorescence from CEN.PK 113-7D. A qualitative estimate of residual glucose was determined using test strips (VWR) at each sampling point.

Vanillin- $\boldsymbol{\beta}$-Glucoside Production Strains. The five-gene vanillin- $\beta$-glucoside biosynthetic pathway (Figure $2 \mathrm{~A}$ ) was introduced using CasEMBLR ${ }^{42}$ into three loci: BGL1/EXG1 and $A D H 6$, to simultaneously knock these genes out and thereby avoid product degradation, ${ }^{29,30}$ and the EasyClone site XII-5. ${ }^{43}$ The pathway was integrated into the genome in two steps. In the first reaction, 4 pmol of each part (insertion homology arms, promoters, terminators, and genes (ACAR and EntD)) were co-transformed with a gRNA expression plasmid targeting ADH6 (clonNAT resistance) into CEN.PK 113-7D already carrying a Cas9 expression plasmid (G418 resistant). Following genotyping of transformants and sequence verification of the assemblies, colonies with correctly assembled and integrated expression cassettes were cured for loss of the $A D H 6$ plasmid and subsequently transformed with a gRNA plasmid targeting EXG1 and site XII-5, along with parts for expression cassettes of OMT, UGT, and 3-DSD. Colonies were screened as done previously, and those containing all five genes of the pathway successfully integrated and assembled were retained for fermentations. Figure S5 gives the layout of the pathway integration and the genotyping assays. The gRNA plasmids are listed in Table S5 in the Supporting Information.

Bioreactor Cultures and HPLC Analysis of Metabolites. For the transcriptome analysis, an overnight culture of $\log$-phase $S$. cerevisiae grown in Delft medium was inoculated into a $500 \mathrm{~mL}$ Delft medium at a starting OD of 0.03 . The cultures were carried out in $1 \mathrm{~L}$ Biostat $\mathrm{Q}$ bioreactors (Sartorius) in triplicate at $30^{\circ}$ with $800 \mathrm{rpm}$ agitation, with controlled aeration and $\mathrm{pH}$ maintained at 6 using $2 \mathrm{M} \mathrm{NaOH}$. Fermentation broth was sampled every $2 \mathrm{~h}$ for the monitoring of OD, and glucose and yeast metabolites by high-performance liquid chromatography (HPLC). For the latter, the broth sample was centrifuged at $10000 \mathrm{~g}$ for $2 \mathrm{~min}$, and the supernatant was syringe-filtered using a $0.22 \mu \mathrm{m}$ syringe filter. These were diluted 2-fold or 5-fold for HPLC analysis (UltiMate 3000, Dionex) as previously described, ${ }^{44}$ and data acquired and analyzed using Chromeleon (Dionex/Thermofisher). For the cultivations of the vanillin- $\beta$-glucoside production strains, a single colony of each of the vanillin- $\beta$ glucoside production strains C-VG, ScASR.V001 and ScASR.V003 were picked from a YPD plate to inoculate a culture in Delft medium. Overnight cultures were used as inoculum to start duplicate cultures of each strain in Delft medium at $\mathrm{pH} 6.0$ in $250 \mathrm{~mL}$ shake flasks with a starting $\mathrm{OD}_{600 \mathrm{~nm}}$ of $\sim 0.2$. Growth rates were calculated based on $\mathrm{OD}_{600 \mathrm{~nm}}$ measurements in the exponential growth phase (see Figure S6 in the Supporting Information). For the metabolite profile of vanillin- $\beta$-glucoside and precursors, the strains were cultivated in duplicates in 24-deep well plates using $5 \mathrm{~mL}$ Delft medium at $\mathrm{pH} 6.0$ (Figure S8). The respective cultures were inoculated at a starting $\mathrm{OD}_{600 \mathrm{~nm}}$ of $\sim 0.04$ with a previously prepared overnight culture, and $\mathrm{OD}_{600 \mathrm{~nm}}$ measured during the course of the cultivation by means of plate reader (SynergyMX). Samples taken at regular time intervals were extracted and quantified by HPLC, as previously described. ${ }^{30}$ For glucose and ethanol quantifications, samples taken during cultivations were centrifuged for $5 \mathrm{~min}$ at $13000 \mathrm{rpm}$ and 4 ${ }^{\circ} \mathrm{C}$. Thereafter, $20 \mu \mathrm{L}$ of the supernatants were analyzed using an Aminex HPX-87H column (Bio-Rad) in an Ultimate 3000 HPLC system (ThermoFisher Scientific) with a refractive index detector (RI-101 Refractive Index Detector, Shodex) (see Figure S7). The column was set at $45^{\circ} \mathrm{C}$ and eluted with $5 \mathrm{mM} \mathrm{H}_{2} \mathrm{SO}_{4}$ at a flow rate of $0.6 \mathrm{~mL} \mathrm{~min}^{-1}$, as previously described. $^{45}$

RNaseq and Transcriptome Data Analysis. Thirty (30) OD units of bioreactor yeast culture grown in 6, 14, and $26 \mathrm{~h}$ were harvested for total RNA extraction, the time points corresponding to at lag/early log phase, mid-log phase ,and late-log phase/diauxic shift. Sampling points were determined from trends in growth curves. Cells were harvested from fermentation broth in chilled $15 \mathrm{~mL}$ centrifuge tubes half-filled with ice, and then the total RNA was extracted, as previously described using the RNeasy Mini kit (Qiagen). ${ }^{46}$ RNA concentration and quality control were performed using a Qubit 2.0 fluorometer (Life Technologies) and an Agilent 2100 Bioanalyzer with RNA 6000 Nano kit, respectively (Agilent). RNaseq was performed as previously described. ${ }^{47}$ TopHat (2.0.14) and the Cufflinks (2.2.1) suite were employed for RNaseq analysis, as described previously, ${ }^{48}$ using the reference genome and annotations for S. cerevisiae S288C (NCBI RefSeq GCF_000146045.2). Three biological replicates were used to determine expression levels (Fragments Per Kilobase of exon per Million fragments mapped; FPKM) for each condition. Upper-quartile normalization was preferred and reads mapping to rRNA genes were masked. Cuffdiff is used to obtain fold change differences and to perform statistical testing. A $q$-value cutoff of $<0.05$ was used to identify genes that have significant differential expression (Table S1). GO and GO slim mappings were retrieved from the Saccharomyces Genome Database (http://www. yeastgenome.org). When extraction was not possible due to chromosome ends, the largest possible sequence fitting the criteria was used. For transcription factor binding site analysis of CSRE, sequences spanning -700 bp to -125 bp upstream from CDS features (or largest possible sequence fitting the criteria) were extracted and patterns matching those listed in Table S2 were identified using biopython (Version 1.68).

Database for RNA-seq Data. RNA-seq data have been deposited in the ArrayExpress database at EMBL-EBI (www. 
ebi.ac.uk/arrayexpress), under accession number E-MTAB7657 (also see Table S1).

\section{ASSOCIATED CONTENT}

\section{S Supporting Information}

The Supporting Information is available free of charge on the ACS Publications website at DOI: 10.1021/acssynbio.9b00027.

Sequences (PDF)

Additional figures, tables, and references cited (PDF)

\section{AUTHOR INFORMATION}

\section{Corresponding Author}

*Tel.: +45 6128 4850. Fax: +45 4525 8001. E-mail: mije@ biosustain.dtu.dk.

\section{ORCID $\odot$}

Konstantin Schneider: 0000-0001-9595-324X

Michael K. Jensen: 0000-0001-7574-4707

\section{Present Address}

${ }^{\#}$ School of Microbiology, University College Cork, Cork, Ireland.

\section{Author Contributions}

A.S.R, M.K.J., and J.D.K. conceived the study. A.S.R. constructed plasmids and strains and performed flow cytometry analyses. A.V.L. and K.S. performed all fermentations, and A.V.L. and J.Q. performed all HPLC measurements. E.Ö. conducted RNaseq analysis. A.S.R., M.K.J., E.Ö., A.V.L., and J.Q. analyzed data. A.S.R. and M.K.J. wrote the manuscript.

\section{Notes}

The authors declare the following competing financial interest(s): J.D.K. has a financial interest in Amyris, Lygos, Demetrix, Constructive Biology, Maple Bio, and Napigen.

\section{ACKNOWLEDGMENTS}

This work was funded by the Novo Nordisk Foundation and 7th Framework program under the European Union (BioREFINE-2G, Project No. FP7-613771). We wish to thank Anna Koza for technical assistance with RNAseq, Signe Stentoft for help with strain construction, Lars Boje Petersen and Charlotte Brochner Olsen for help with HPLC, and Inger Rosenstadt for help with the bioreactor culture.

\section{REFERENCES}

(1) Paddon, C. J., Westfall, P. J., Pitera, D. J., Benjamin, K., Fisher, K., McPhee, D., Leavell, M. D., Tai, A., Main, A., Eng, D., et al. (2013) High-Level Semi-Synthetic Production of the Potent Antimalarial Artemisinin. Nature 496 (7446), 528-532.

(2) Brown, S., Clastre, M., Courdavault, V., and O'Connor, S. E. (2015) De Novo Production of the Plant-Derived Alkaloid Strictosidine in Yeast. Proc. Natl. Acad. Sci. U. S. A. 112 (11), $3205-3210$.

(3) Kirby, J., Dietzel, K. L., Wichmann, G., Chan, R., Antipov, E., Moss, N., Baidoo, E. E. K., Jackson, P., Gaucher, S. P., Gottlieb, S., et al. (2016) Engineering a Functional 1-Deoxy-D-Xylulose 5Phosphate (DXP) Pathway in Saccharomyces Cerevisiae. Metab. Eng. 38, 494-503.

(4) Glick, B. R. (1995) Metabolic Load and Heterologous Gene Expression. Biotechnol. Adv. 13 (2), 247-261.

(5) Solomon, K. V., and Prather, K. L. J. (2011) The Zero-Sum Game of Pathway Optimization: Emerging Paradigms for Tuning. Biotechnol. J. 6 (9), 1064-1070.
(6) Heyland, J., Blank, L. M., and Schmid, A. (2011) Quantification of Metabolic Limitations during Recombinant Protein Production in Escherichia Coli. J. Biotechnol. 155 (2), 178-184.

(7) Peng, B., Williams, T. C., Henry, M., Nielsen, L. K., and Vickers, C. E. (2015) Controlling Heterologous Gene Expression in Yeast Cell Factories on Different Carbon Substrates and across the Diauxic Shift: A Comparison of Yeast Promoter Activities. Microb. Cell Fact. 14, 91.

(8) Lee, K. M., and DaSilva, N. A. (2005) Evaluation of the Saccharomyces Cerevisiae ADH2 Promoter for Protein Synthesis. Yeast 22 (6), 431-440.

(9) Partow, S., Siewers, V., Bjørn, S., Nielsen, J., and Maury, J. (2010) Characterization of Different Promoters for Designing a New Expression Vector in Saccharomyces Cerevisiae. Yeast 27 (11), 955964

(10) Cao, L., Tang, X., Zhang, X., Zhang, J., Tian, X., Wang, J., Xiong, M., and Xiao, W. (2014) Two-Stage Transcriptional Reprogramming in Saccharomyces Cerevisiae for Optimizing Ethanol Production from Xylose. Metab. Eng. 24, 150-159.

(11) Weinhandl, K., Winkler, M., Glieder, A., and Camattari, A. (2014) Carbon Source Dependent Promoters in Yeasts. Microb. Cell Fact. 13, 5 .

(12) Wang, J., Zhai, H., Rexida, R., Shen, Y., Hou, J., and Bao, X. Developing Synthetic Hybrid Promoters to Increase Constitutive or Diauxic Shift-Induced Expression in Saccharomyces Cerevisiae. FEMS Yeast Res. 2018, 18 (8) DOI: 10.1093/femsyr/foy098.

(13) Du, J., Yuan, Y., Si, T., Lian, J., and Zhao, H. (2012) Customized Optimization of Metabolic Pathways by Combinatorial Transcriptional Engineering. Nucleic Acids Res. 40 (18), e142.

(14) Rajkumar, A. S., Liu, G., Bergenholm, D., Arsovska, D., Kristensen, M., Nielsen, J., Jensen, M. K., and Keasling, J. D. (2016) Engineering of Synthetic, Stress-Responsive Yeast Promoters. Nucleic Acids Res. 44 (17), e136.

(15) Cheng, J. K., and Alper, H. S. (2016) Transcriptomics-Guided Design of Synthetic Promoters for a Mammalian System. ACS Synth. Biol. 5 (12), 1455-1465.

(16) Shi, S., Choi, Y. W., Zhao, H., Tan, M. H., and Ang, E. L. (2017) Discovery and Engineering of a 1-Butanol Biosensor in Saccharomyces Cerevisiae. Bioresour. Technol. 245, 1343-1351.

(17) Turcotte, B., Liang, X. B., Robert, F., and Soontorngun, N. (2010) Transcriptional Regulation of Nonfermentable Carbon Utilization in Budding Yeast. FEMS Yeast Res. 10 (1), 2-13.

(18) Roth, S., Kumme, J., and Schüller, H.-J. (2004) Transcriptional Activators Cat8 and Sip4 Discriminate between Sequence Variants of the Carbon Source-Responsive Promoter Element in the Yeast Saccharomyces Cerevisiae. Curr. Genet. 45 (3), 121-128.

(19) Hiesinger, M., Roth, S., Meissner, E., and Schüller, H. J. (2001) Contribution of Cat8 and Sip4 to the Transcriptional Activation of Yeast Gluconeogenic Genes by Carbon Source-Responsive Elements. Curr. Genet. 39 (2), 68-76.

(20) Soontorngun, N., Larochelle, M., Drouin, S., Robert, F., and Turcotte, B. (2007) Regulation of Gluconeogenesis in Saccharomyces Cerevisiae Is Mediated by Activator and Repressor Functions of Rds2. Mol. Cell. Biol. 27 (22), 7895-7905.

(21) Walther, K., and Schüller, H. J. (2001) Adr1 and Cat8 Synergistically Activate the Glucose-Regulated Alcohol Dehydrogenase Gene $\mathrm{ADH} 2$ of the Yeast Saccharomyces Cerevisiae. Microbiology 147, 2037-2044.

(22) Mercado, J. J., and Gancedo, J. M. (1992) Regulatory Regions in the Yeast FBP1 and PCK1 Genes. FEBS Lett. 311 (2), 110-114.

(23) Soontorngun, N., Baramee, S., Tangsombatvichit, C., Thepnok, P., Cheevadhanarak, S., Robert, F., and Turcotte, B. (2012) GenomeWide Location Analysis Reveals an Important Overlap between the Targets of the Yeast Transcriptional Regulators Rds2 and Adr1. Biochem. Biophys. Res. Commun. 423 (4), 632-637.

(24) Bitter, G. A., Chang, K. K., and Egan, K. M. (1991) A MultiComponent Upstream Activation Sequence of the Saccharomyces Cerevisiae Glyceraldehyde-3-Phosphate Dehydrogenase Gene Promoter. Mol. Gen. Genet. 231 (1), 22-32. 
(25) Chambers, A., Packham, E. A., and Graham, I. R. (1995) Control of Glycolytic Gene Expression in the Budding Yeast (Saccharomyces Cerevisiae). Curr. Genet. 29 (1), 1-9.

(26) Uemura, H., Koshio, M., Inoue, Y., Lopez, M. C., and Baker, H. V. (1997) The Role of Gcrlp in the Transcriptional Activation of Glycolytic Genes in Yeast Saccharomyces Cerevisiae. Genetics 147 (2), $521-532$.

(27) Zhang, N., Wu, J., and Oliver, S. G. (2009) Gis1 Is Required for Transcriptional Reprogramming of Carbon Metabolism and the Stress Response during Transition into Stationary Phase in Yeast. Microbiology 155 (5), 1690-1698.

(28) Scott, E. W., and Baker, H. V. (1993) Concerted Action of the Transcriptional Activators REB1, RAP1, and GCR1 in the High-Level Expression of the Glycolytic Gene TPI. Mol. Cell. Biol. 13 (1), 543550.

(29) Hansen, E. H., Møller, B. L., Kock, G. R., Bünner, C. M., Kristensen, C., Jensen, O. R., Okkels, F. T., Olsen, C. E., Motawia, M. S., and Hansen, J. (2009) De Novo Biosynthesis of Vanillin in Fission Yeast (Schizosaccharomyces Pombe) and Baker's Yeast (Saccharomyces Cerevisiae). Appl. Environ. Microbiol. 75 (9), 2765-2774.

(30) Strucko, T., Magdenoska, O., and Mortensen, U. H. (2015) Benchmarking Two Commonly Used Saccharomyces Cerevisiae Strains for Heterologous Vanillin- $\beta$-Glucoside Production. Metabolic Engineering Communications 2, 99-108.

(31) Brochado, A. R., Matos, C., Møller, B. L., Hansen, J., Mortensen, U. H., and Patil, K. R. (2010) Improved Vanillin Production in Baker's Yeast through in Silico Design. Microb. Cell Fact. 9, 84.

(32) Strucko, T., Buron, L. D., Jarczynska, Z. D., Nødvig, C. S., Mølgaard, L., Halkier, B. A., and Mortensen, U. H. (2017) CASCADE, a Platform for Controlled Gene Amplification for High, Tunable and Selection-Free Gene Expression in Yeast. Sci. Rep. 7, 41431.

(33) Wang, Z., Bai, X., Guo, X., and He, X. (2017) Regulation of Crucial Enzymes and Transcription Factors on 2-Phenylethanol Biosynthesis via Ehrlich Pathway in Saccharomyces Cerevisiae. J. Ind. Microbiol. Biotechnol. 44 (1), 129-139.

(34) Leavitt, J. M., Tong, A., Tong, J., Pattie, J., and Alper, H. S. (2016) Coordinated Transcription Factor and Promoter Engineering to Establish Strong Expression Elements in Saccharomyces Cerevisiae. Biotechnol. J. 11 (7), 866-876.

(35) Rantasalo, A., Kuivanen, J., Penttilä, M., Jäntti, J., and Mojzita, D. (2018) Synthetic Toolkit for Complex Genetic Circuit Engineering in Saccharomyces Cerevisiae. ACS Synth. Biol. 7 (6), 1573-1587.

(36) Jongedijk, E., Cankar, K., Buchhaupt, M., Schrader, J., Bouwmeester, H., and Beekwilder, J. (2016) Biotechnological Production of Limonene in Microorganisms. Appl. Microbiol. Biotechnol. 100 (7), 2927-2938.

(37) Westfall, P. J., Pitera, D. J., Lenihan, J. R., Eng, D., Woolard, F. X., Regentin, R., Horning, T., Tsuruta, H., Melis, D. J., Owens, A., et al. (2012) Production of Amorphadiene in Yeast, and Its Conversion to Dihydroartemisinic Acid, Precursor to the Antimalarial Agent Artemisinin. Proc. Natl. Acad. Sci. U. S. A. 109 (3), E111-E118.

(38) Curran, K. A., Crook, N. C., Karim, A. S., Gupta, A., Wagman, A. M., and Alper, H. S. (2014) Design of Synthetic Yeast Promoters via Tuning of Nucleosome Architecture. Nat. Commun. 5, 4002.

(39) Lubliner, S., Regev, I., Lotan-Pompan, M., Edelheit, S., Weinberger, A., and Segal, E. (2015) Core Promoter Sequence in Yeast Is a Major Determinant of Expression Level. Genome Res. 25 (7), 1008-1017.

(40) Portela, R. M. C., Vogl, T., Kniely, C., Fischer, J. E., Oliveira, R., and Glieder, A. (2017) Synthetic Core Promoters as Universal Parts for Fine-Tuning Expression in Different Yeast Species. ACS Synth. Biol. 6 (3), 471-484.

(41) Shabbir Hussain, M., Gambill, L., Smith, S., and Blenner, M. A. (2016) Engineering Promoter Architecture in Oleaginous Yeast Yarrowia Lipolytica. ACS Synth. Biol. 5 (3), 213-223.

(42) Jakočiūnas, T., Rajkumar, A. S., Zhang, J., Arsovska, D., Rodriguez, A., Jendresen, C. B., Skjødt, M. L., Nielsen, A. T.,
Borodina, I., Jensen, M. K., and Keasling, J. D. (2015) CasEMBLR: Cas9-Facilitated Multiloci Genomic Integration of in Vivo Assembled DNA Parts in Saccharomyces Cerevisiae. ACS Synth. Biol. 4, 12261234.

(43) Jensen, N. B., Strucko, T., Kildegaard, K. R., David, F., Maury, J., Mortensen, U. H., Forster, J., Nielsen, J., and Borodina, I. (2014) EasyClone: Method for Iterative Chromosomal Integration of Multiple Genes in Saccharomyces Cerevisiae. FEMS Yeast Res. 14 (2), 238-248.

(44) Stovicek, V., Borodina, I., and Forster, J. (2015) CRISPR-Cas System Enables Fast and Simple Genome Editing of Industrial Saccharomyces Cerevisiae Strains. Metabolic Engineering Communications 2, 13-22.

(45) Qin, J., Zhou, Y. J., Krivoruchko, A., Huang, M., Liu, L., Khoomrung, S., Siewers, V., Jiang, B., and Nielsen, J. (2015) Modular Pathway Rewiring of Saccharomyces Cerevisiae Enables High-Level Production of L-Ornithine. Nat. Commun. 6, 8224.

(46) Kildegaard, K. R., Hallström, B. M., Blicher, T. H., Sonnenschein, N., Jensen, N. B., Sherstyk, S., Harrison, S. J., Maury, J., Herrgård, M. J., Juncker, A. S., et al. (2014) Evolution Reveals a Glutathione-Dependent Mechanism of 3-Hydroxypropionic Acid Tolerance. Metab. Eng. 26, 57-66.

(47) Skjoedt, M. L., Snoek, T., Kildegaard, K. R., Arsovska, D., Eichenberger, M., Goedecke, T. J., Rajkumar, A. S., Zhang, J., Kristensen, M., Lehka, B. J., et al. (2016) Engineering Prokaryotic Transcriptional Activators as Metabolite Biosensors in Yeast. Nat. Chem. Biol. 12 (11), 951-958.

(48) Trapnell, C., Roberts, A., Goff, L., Pertea, G., Kim, D., Kelley, D. R., Pimentel, H., Salzberg, S. L., Rinn, J. L., and Pachter, L. (2012) Differential Gene and Transcript Expression Analysis of RNA-Seq Experiments with TopHat and Cufflinks. Nat. Protoc. 7 (3), 562-578. 